\title{
Resource Reservation in Wireless Networks based on Pattern Recognition
}

\author{
W. W. H. Yu, Changhua He
}

\begin{abstract}
Resource Reservation is very important for handoff control in wireless networks. Now many researches have aimed to predict the user's destination cell based on its movement pattern for efficient resource reservation. In the future networks with small size cells, handoffs will occur more frequently and the user's movement will be more like random processes, so it is not practical to predict the accurate destination of a user. We propose a statistical strategy for resource reservation through the estimation of a user's Transfer Probabilities, which represent the possibilities of the user leaving the current cell and entering the neighboring cells. The resources reserved for a user in each base station are proportional to the user's Transfer Probabilities. A mathematical model is proposed to obtain the Transfer Probabilities of a user from the initial states (position, velocity and direction) through simulation of the user's movement. Neural Networks are developped to predict the Transfer Probabilities of a user from the initial states and facilitate efficient resource reservation.
\end{abstract}

\section{Introduction}

Wireless multimedia networks will adopt the topology of small size cells to increase the system capacity in the future, which will lead to frequent handoffs while users move among cells remaining connected to the base stations. During the handoff process, resources are reserved and allocated to the user as quickly as possible to avoid possible call dropping or decreasing QoS (Quality of Service). Because of the inherent limitation of resources in wireless environments and the large requirement of resources in multimedia applications, excessive resource reservation will reduce the system capacity and affect QoS significantly. So it is necessary to develop an adaptive algorithm to provide efficient resource reservation and guarantee QoS. Obviously, if accurate knowledge about the user's trajectory prior to its movement is known, the system can plan resource reservation most efficiently by reserving only the required resources in the cells along the user's route.

However, in the real situation, the user's intended route is generally unknown to the system. Many researchers have studied ways to predict the user's route correctly based on the user's movement pattern. Liu and Maguire Jr. [2] developed a two-layer model which describes a user's behavior as repetitions of some elementary movement patterns stored in a user's profile. One layer is the regular movement namely Movement Circle (MC) and Movement Track (MT), which is based on the assumption that wherever a user moves from, it will eventually return or has returned. This introduces many different circle-like patterns in the model. The other layer is random movement, namely Markov Chain Model, which only represents the behavior of random parts in the user movement. Simulations have shown that the prediction efficiency is about $95 \%$. This is a fairly good result, but it is very sensitive to the random factor, which represents random parts of the movement.

Liu et al [3] suggested another two-layer hierarchy model for the user movement prediction. The top layer is the Global Mobility Model, whose resolution is in terms of cells crossed by the user during the whole lifetime. This is a deterministic model created for inter-cell movements. The bottom layer is the Local Mobility Model, whose resolution is in terms of a 3-tuple sample space (velocity, direction and position) that varies with time. This is a stochastic model with dynamically changing state variables in order to simulate intra-cell movements. The authors predicted the destination of the user with a certain position and velocity, reserved resources in those base stations around the destination. Finally they obtained accurate results in handoff control and greatly reduced network congestion. Also there is another model used to predict the user's next destination with artificial Neural Networks [8].

All these models assume the user's movement can be classified using mathematical processes. Of course these are more appropriate for the approximation of movements among large size cells than small size cells, where handoffs occur more frequently, the user's behaviors are more like random processes, and the prediction from any user profiles will not be effective.

Fortunately, the user's current movement pattern can provide useful information about the user's possible movement in the near future because generally the user doesn't change its movement abruptly. Based on the idea, Levine et al [5] proposed a concept of shadow cluster, in which each user has its own specific area called a shadow, which moves along with the user. Each base station in the shadow cluster anticipates the user's arrival and reserves resources for it. The number of base stations that reserve resources will determine the overall system efficiency and QoS guarantee. In order to introduce more adaptivity, Jelena Misic et al [4] proposed a new distributed admission control strategy in which the calculation of the estimated 
number of resources needed to accommodate handoffs are triggered by certain events instead of being calculated at call admissions at regular time intervals. The events that trigger the re-calculation are handoff, origination and termination of calls in the neighborhood of the target cell. The probability that a call from a given originating cell will visit the target cell within its lifetime will be referred to as the Spatial Activity Factor of the call. The SAF of a call in any target cell depends on the relative distance of the target cell from the originating cell, the distributions of the call duration time and the call dwell time, and the directional characteristics of the terrain. These algorithms are useful for reducing the number of reserved resources even in random movements, but almost impossible to implement in general environment because of the associated complexity. The features aren't easy to obtain in the real communications such as call duration time, call dwell time and so on.

In this paper, we construct a model to describe the user's random movement in small size cells, and extract the user's velocity, direction and location as the most important features of its movement, and classify the user's Transfer Probabilities based on these features, which represent the possibilities the user leaving the current cell and entering one of its neighboring cells. Resources are reserved among all its neighbor cells proportional to the value of Transfer Probabilities. Back Propagation Neural Networks are used to approximate the relationship between the user's initial states and its Transfer Probabilities.

The rest of the paper is divided into the following sections: Section 2 states a random model about the user's movement in small size cells. Section 3 proposes the neural network model. Section 4 describes the detailed algorithm for resource reservation. Section 5 shows the simulation results and discussions, and Section 6 draws the conclusion for our work.

\section{Movement Model}

In the wireless systems with small size cells, the user's movements are more random-like, and more likely to cross the cell boundaries frequently. As a result, it is more difficult to predict the destination cell, which is dependent on the entire user's path. Some variables that characterize the user's movement such as the initial location, the direction of travel and the velocity can still provide useful information about its possible movement. The initial location of a user in the current cell determines which cell is the nearest to the user among all its neighboring cells. The velocity and direction can determine the user's possible movement in the near future. Therefore, these variables indicate the probability that the user finally arrive at a cell.

The probability of a user leaving the current cell and entering a neighboring cell is the user's Transfer Probability to this neighboring cell. Instead of predicting the user's next destination cell from its current movement pattern, calculating the Transfer Probability Vector is considered a much better approach to estimating the number of reserved resources in each base station because sometimes it is very hard to specify a destination cell from several cells for which a user has almost the same Transfer Probabilities. In order to achieve the relationship between the user's initial states and its Transfer Probabilities, the user's movement pattern should be studied.

It is very difficult to generate a truly random route to simulate the random nature in reality. An approximation of the user's movement assumes that all the users move with a constant velocity $v_{0}$ and in a direction $Q_{0}$ for a short time interval $\tau$ after generating a call in a cell at an initial location $P\left(x_{0}, y_{0}\right)$. At the end of the time interval $\tau$, the user will arrive at a new location $P\left(x_{1}, y_{1}\right)$. The velocity and the direction at the new location are incremented by $d v_{l}$ and $d Q_{I}$ respectively. The user continues moving with the new velocity $v_{1}=v_{0}+d v_{1}$ and in the direction $Q_{1}=Q_{0}+d Q_{1}$ for another time interval $\tau$. The same process repeats in a series of iteration at a time interval of $\tau$. At the end of the $k^{\text {th }}$ iteration, the total time duration of travel is $T_{k}=k \tau$. Figure 1 illustrates the process of the user's movement.

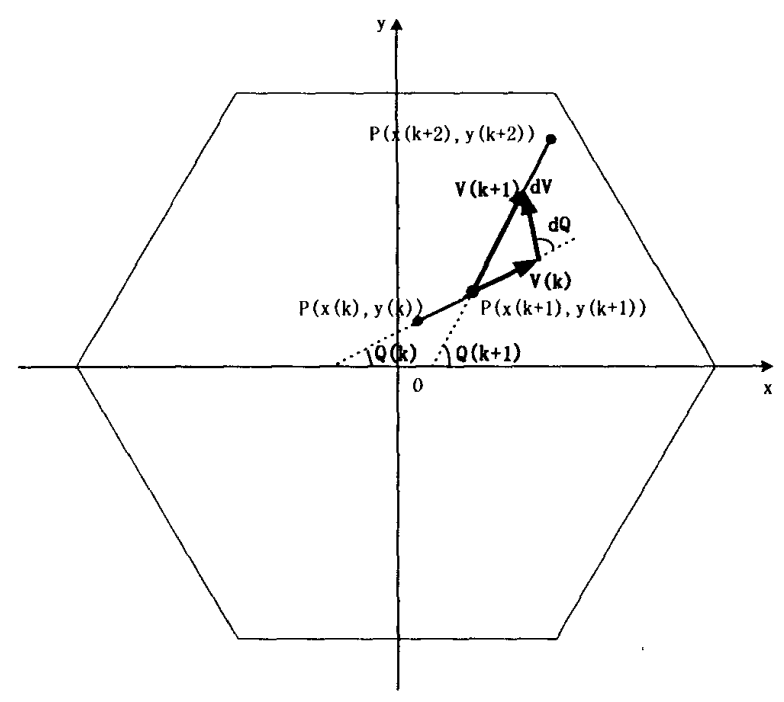

Figure 1: Movement Model

At time $T_{k}=k \tau$, the user is at the position $P\left(x_{k}, y_{k}\right)$. After a time interval $\tau$, or at time $T_{k+1}=(k+1) \tau$, it moves to the new position $P\left(x_{k+1}, y_{k+1}\right)$. The speed is changed 
from $\left(v_{k}, Q_{k}\right)$ to $\left(v_{k+1}, Q_{k+1}\right)$ based on a randomly generated difference $(d v, d Q)$. After another time interval $\tau$, it will move to another position $P\left(x_{k+2}, y_{k+2}\right)$ and repeat the same process. The relationship between the user's states at time $T_{k+1}=(k+1) \tau$ and $T_{k}=k \tau$ can be derived as below:

$$
\begin{aligned}
& x_{k+1}=x_{k}+\tau * v_{k} * \cos Q_{k} \\
& y_{k+1}=y_{k}+\tau * v_{k} * \sin Q_{k} \\
& v_{k+1}=\sqrt{v_{k}^{2}+d v^{2}-2 * d v * v_{k} * \cos (\pi-d Q)} \\
& Q_{k+1}=Q_{k}+\arccos \frac{v_{k+1}^{2}+v_{k}^{2}-d v^{2}}{2 * v_{k} * v_{k+1}}, 0 \leq d Q<\pi \\
& Q_{k+1}=Q_{k}-\arccos \frac{v_{k+1}^{2}+v_{k}^{2}-d v^{2}}{2 * v_{k} * v_{k+1}}, \pi \leq d Q<2 \pi
\end{aligned}
$$

With these equations, the whole route of a user can be obtained in iteration steps based on the initial states and the randomly generated incremental velocity $d v$ and angle of direction $d Q$. The length of time interval $\tau$ will determine the degree of randomness of the movement. The smaller the time interval is, the more the user's movement resembles a truly random movement in reality.

For users with each set of initial states, it will move according to above model for many times, and each route ends when the user enters one of its neighboring cells. After thousands of times of iterations for a user with the same initial states, its Transfer Probabilities can be obtained through calculating the times the user enters each neighboring cell respectively. Based on the knowledge obtained from above model, Neural Networks can be trained to recognize the Transfer Probabilities for a user's initial states.

\section{Neural Networks Model}

In the proposed neural networks, Back Propagation algorithm is adopted to train the network with the sample data obtained from above model. As a result, the Neural Networks System is constructed as shown in Figure 2. Four inputs are the user's initial states, and six outputs are the corresponding Transfer Probability Vector. The network. has two hidden layers and 30 nodes in each layer respectively. The non-linear functions of each node are all sigmoid logistic functions and the connection weights are stored in a matrix.

All the results for neural networks with different topologies, say different number of hidden layers and different number of nodes in each hidden layer, are listed in Figure 3, 4, 5 and 6. Obviously, two hidden layers are necessary to achieve a high precision for this problem, and
$30 \times 30$ nodes in each hidden layer are enough considering both the precision and the complexity.

In our implementation, 1000 samples are obtained from the model in Section 2, among which 800 samples are used to train the network and 200 samples are used to test the network. For each testing sample, square errors of the actual output and desired output are calculated and drawn in Figure 7. It shows the network can achieve rather accurate results about Transfer Probabilities for most inputs of the initial states in the testing samples.

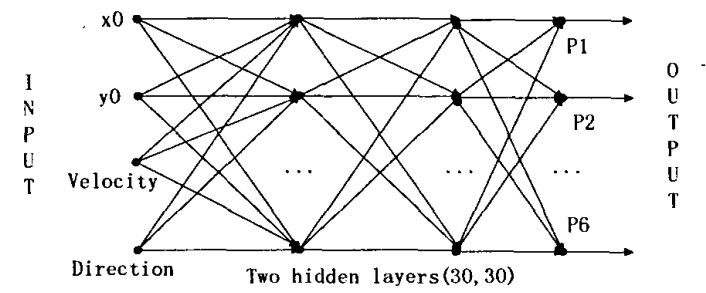

Figure 2: Neural Networks System

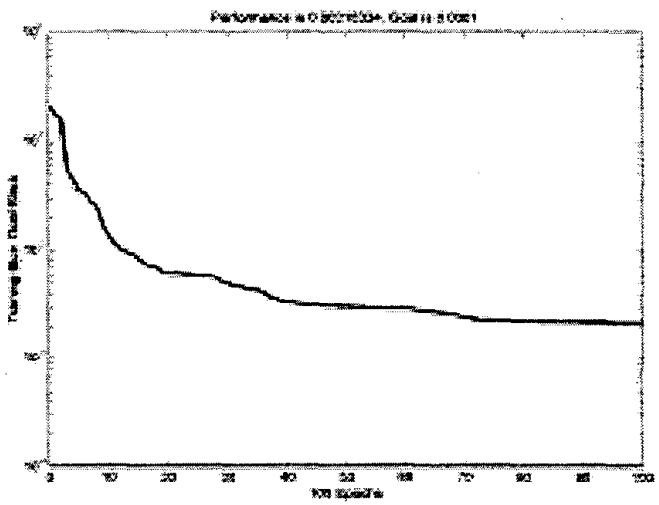

Figure 3: NN with One hidden layer and 20 nodes

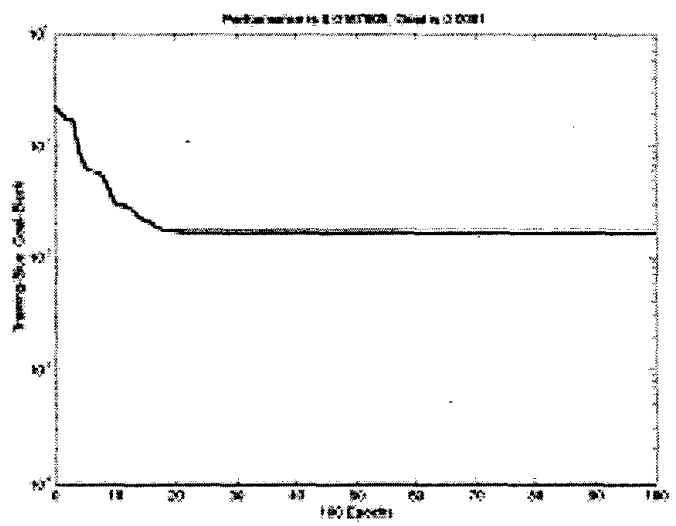

Figure 4: NN with One hidden layer and 50 nodes 


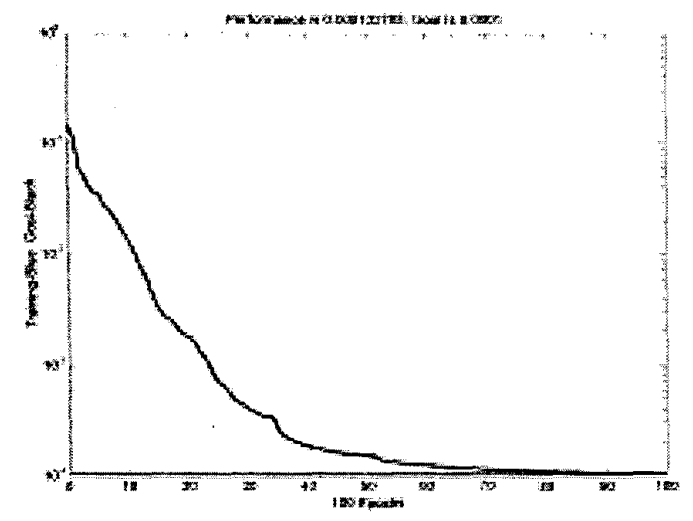

Figure 5: $\mathrm{NN}$ with Two hidden layers and $20 \times 20$ nodes

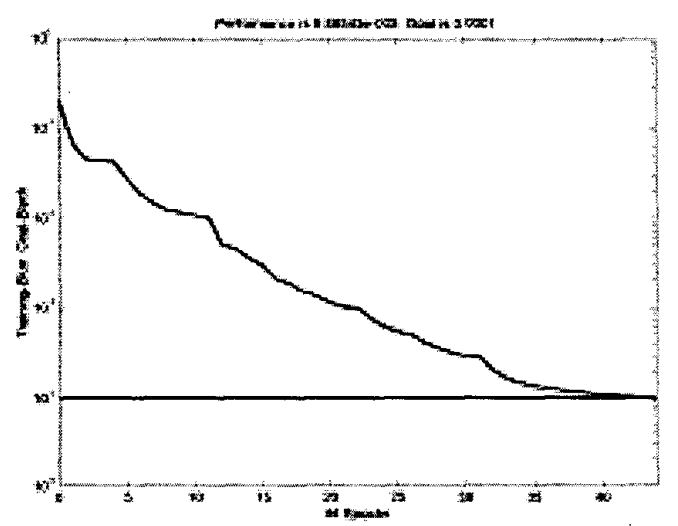

Figure 6: NN with Two hidden layers and $30 \times 30$ nodes

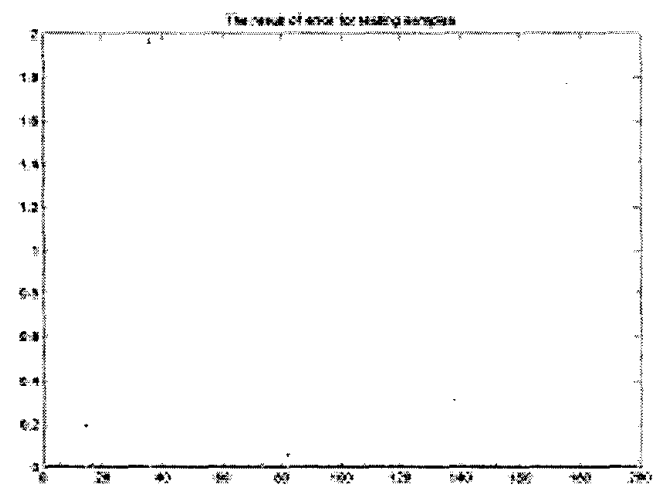

Figure 7: Testing Results for 200 samples

Although the training time is very long for Neural Networks (about 8 hours in our simulation), the execution for each input vector is very fast after training. So we can train the networks off-line, and implement them in the real systems after training. During our simulation, the execute time for each input vector of the network is in the order of $m s$, which satisfies the requirement of real time communication, and the error rate is lower than $10^{-4}$.

\section{Resource Reservation}

Once the topology of Neural Networks is determined, it can be used to achieve the Transfer Probabilities based on the user's initial states. Thus resources can be reserved according to the estimation of Transfer Probabilities [1]. For a systems with hexagonal cells, $P(i, j, k, t)$ is the Transfer Probability of the user user $j$ in cell $i$ to neighboring cell $k$ at time $t$, where $k \in S_{i}$ and $S_{i}$ is the set of all the neighboring cells of the current cell $i$. Assuming $r(i, j)$ is the total resources required to achieve QoS guarantee, $R(i, j, k, t)$ is the resources reserved in the neighboring cell $k$ for such user at time $t$. According to the basic concept of the proposed resources reservation scheme, the reserved resources for the user in each neighboring base station are given by:

$$
R(i, j, k, t)=P(i, j, k, t) \times r(i, j)
$$

The detailed algorithm is described as follows. Each base station has its own reservation pool. When a user becomes active in a new cell with a certain initial states, its current base station will calculate the Transfer Probability Vector through Neural Networks and exchange the information with other neighboring base stations. Then, each of the neighboring base stations will add the number of reserved resources for this user to the reservation pool respectively, according to above equation. When a handoff request is received, the base station will check the reservation pool. If there are enough reserved resources for the handoff, it will allocate the required resources to the user from its reservation pool and calculate the Transfer Probability Vector again. Based on such information, its new neighboring base stations will re-calculate the number of reserved resources for the future handoffs. If there are insufficient resources in the reservation pool, the request will be denied and the call is dropped. When a user breaks connection, the base station will notify all the neighbors to release the resources reserved for it according to its Transfer Probability Vector. In the algorithm, the total reserved resources for handoff requirements are the sum of resources required by all active users in the system.

There may be a concern that the fractional resources reserved for all the users may not be sufficient to facilitate all the hand-offs when the many users eventually enter the cell. While this is theoretically possible, the probability is very small, because not all the users will enter the same cell at the same time and user leaving one cell will not all enter the same cell. Therefore the proposed scheme of reserve a 
fraction of the required resource for one user should not present any real problems. It will be a question of how large the fraction should be to guarantee the QoS level.

The population of active users in each cell is a function of time. Therefore the Transfer Probability must be updated periodically. Each time the Transfer Probability is updated, and information exchanges between adjacent cells take place. Frequent updates introduce a high traffic load and will affect the performance of the network. The strategy should be that the updating of the Transfer Probability should be done periodically and less frequently. A reasonable approach is to have an update only when a user crosses the boundary of two cells when the handoff occurs.

\section{Simulation Results and Discussions}

The simulation is performed among a cell cluster of nineteen hexagonal cells, which circle the center cell layer by layer and has a "wrap-around" topology. That means, when a user exits the cluster boundary, say at the point in the east side, it will enter the cluster from the related point in the west side of the boundary. This assures the simulation is running in a close system, and each cell has six neighboring cells so that no user will move out of the cluster and become unknown to the system. Each cell has its own coordinate axis, when a user goes out of the current cell and handoffs to a new one, its states will be transformed to the new coordinate axis.

The simulation system consists of two control parts: one is resource reservation and the other is call admission control. In our simulation, handoff call requests are given a higher priority to new call requests, because it is less desirable for a user to terminate an ongoing call than to block a new call. Simulation is set up as follows.

1. Cell Radium $r=500 m$.

2. Each cell has a limit of resources 660 , and each call initialized by a user has a normal distribution which has an average time 60s;

3. The population of new users generated in each cell is a uniform distribution in $[0,10]$, and the resource requirement of each user is a uniform distribution in $[1,10]$;

4. New users are generated randomly with the initial positions $P\left(x_{0}, y_{0}\right)$ uniformly distributed in the cell, and the velocity $V_{0}$ is a normal distribution with a mean $6 \mathrm{~km} / \mathrm{h}$ and variance $4(\mathrm{~km} / \mathrm{h})^{2}$, and direction $Q_{0}$ is a uniform distribution in [0,2 $\pi$ ];

5. Each user moves from the current position to a new position with a constant speed during a short time interval $\tau=5 \mathrm{~s}$. At the end of the time interval, the speed will change with the acceleration $d V$, which is a uniform distribution in $[0 ; 2 \mathrm{~km} / \mathrm{h}]$, and the direction difference $d Q$, which obeys a uniform distribution in $[0,2 \pi]$;

6. Each user keeps moving by iterate step 5 until it leaves the current cell and enters one of its neighboring cells. At that time, handoff request is sent to the base station;

7. At any time interval, all handoff requests and new call requests are managed by the call admission control algorithm. If the requests are accepted, the resouce reservation algorithm will be performed to reserve resources in related base stations.

During the simulation, three parameters are considered to test whether the proposed resource reservation strategy is suitable for real situations: Handoff Call Dropping Rate (HCDR), which is the ratio of the number of failed handoffs to the total number of handoff requests; New Call Blocking Rate (NCBR), which is the ratio of the number of denied new calls to the total number of new call requests; And Resource Reservation Efficiency (RRE), which will be calculated as the ratio of the number of resources used during handoff process to the total number of reserved resources. The results are listed in Figure 8, 9 and 10.

In the reservation strategy, although not all the required resources are reserved in a specific cell for a potential handoff user, the result of successful handoffs is satisfactory. It shows, no handoff drops during the whole simulation time. This is because higher priority is given to handoff calls upon new calls. NCBR is a little high at the beginning of simulation, but when the system runs to balance, it is kept around 0.1 , which is acceptable in a heavy traffic. There is a tradeoff between HCDR and NCBR. When the system can tolerate higher dropping handoffs, more new call will be admitted to enter the system. Resource Reservation Efficiency reaches about 0.9 and generally kept upon 0.7 when the system comes to balance, which is very satisfactory with no handoff drops.

\section{Conclusions}

In the future wireless networks, resource reservation will be more and more important because handoff will become more and more frequent in small size cells, and the resources will be more and more limited for multimedia applications.

In this paper, we propose a resource reservation strategy in wireless networks with small size cells. Instead of predicting the destination cell as in other general reservation strategies, we use Transfer Probabilities to guide reservation. The reserved resources in each base station are proportional to the user's Transfer Probabilities. 
In order to obtain accurate value of Transfer Probabilities, we construct a movement model to study the relationship between the user's initial states and its Transfer Probabilities. After that, a Neural Network system is introduced to approximate the relationship after training with the samples obtained from the movement model. Neural Networks is more flexible and accurate comparing with other complex mathematical models, and it can be implemented for different situations with different training samples.

The simulation result shows such strategy is very successful in achieving high resource efficiency within an acceptable level of handoff call dropping rate and new call blocking rate.

As a whole, the algorithm of resource reservation based on Neural Networks is very easy to implement and adaptable for different situations. It offers accurate classification about the user's random movement in small size cells and improved resource efficiency when resources are limited in wireless systems. That means, the system with a fixed number of resources can increase the capacity with such algorithm, which will be the bottleneck in the wireless multimedia networks.

\section{References}

[1] W.W.H. Yu, Changhua He, "A Statistical Strategy of Resource Reservation for Handoff Control in Wireless ATM Networks", Proceedings of the $7^{\text {th }}$ international workshop on Mobile Multimedia Communications (MoMuC2000). Tokyo, Japan, October 2000.

[2] George Y. Liu and Gerald Q. Maguire Jr., "A predictive Mobility Management Algorithm for Wireless Mobile Computing and Communications" Universal Personal Communications, Fourth International IEEE Conference pp. 268-272, 1995.

[3] T. Liu, P. Bahl and I. Chlamtac, "Mobility Modeling, Location Tracking, and Trajectory Prediction in Wireless ATM Networks", IEEE journal on selected areas in communications, vol.16, No.6 pp. 922-936, Aug. 1998.

[4] Jelena Misic, S. T. Chanson and F. S. Lai, "Admission Control for Wireless Networks with Heterogeneous Traffic using Event Based Resource Estimation", Proceedings of the Sixth International Conference on Computer Communications and Networks, Page(s): 262 -269, 1997.

[5] D.A.Levine, I.F.Akyildiz, and M.Naghshineh, "The shadow cluster concept for resource allocation and call admission in ATMbased wireless networks" in Proc. $I^{\text {st }}$ ACM Mobicom, Nov.1995, pp. 142-150; also, IEEE/ACM Trans. Networking, vol.6, pp. 1-10, Feb. 1997.

[6] S. Haykin, "Neural Networks: A Comprehensive Foundation", Upper Sadder River, N. J. : Prentice Hall : New York: Institute of Electrical and Electronica Engineers, c1999.

[7] Richard P. Lippmann, "An Introduction to Computing with Neural Nets", Reprinted from IEEE ASSP Magazine, pp. 4-22, April 1987.

[8] Mahboubeh Lohi, K.Madani, D. Weerakoon and A.H.Aghvami, "Using artificial Neural Networks to improve performance in a wireless ATM network", Vihicular Technology Conference, IEEE $49^{\text {th }}$, Vol. 3, pp. 2029-2033, 1999.

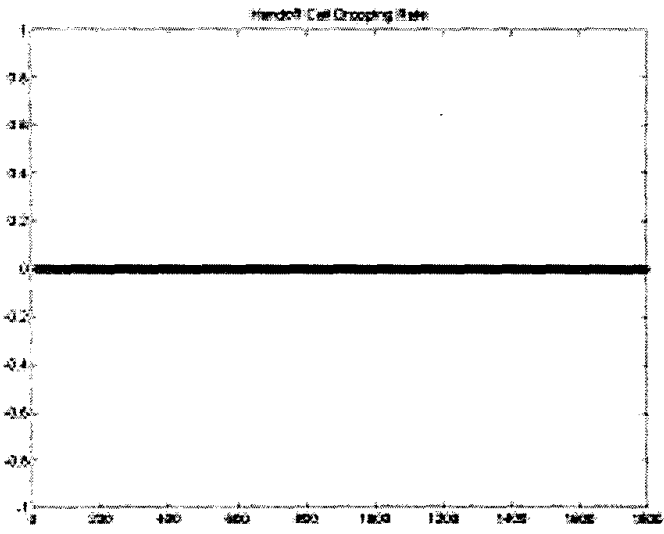

Figure 8: Handoff Call Dropping Rate

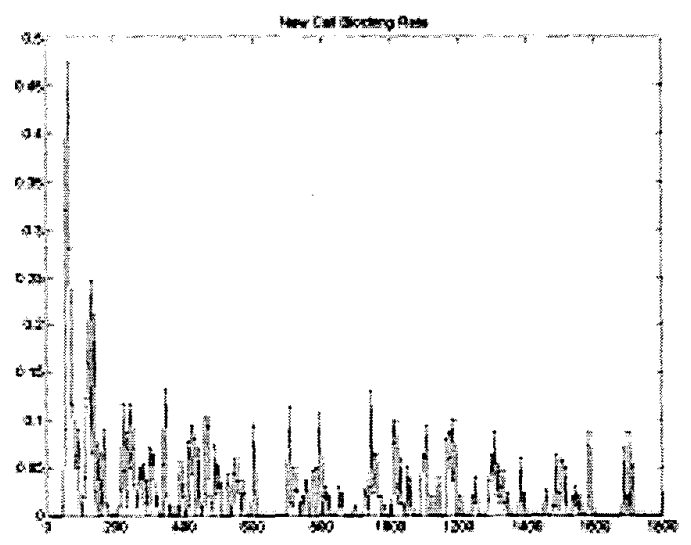

Figure 9: New Call Blocking Rate

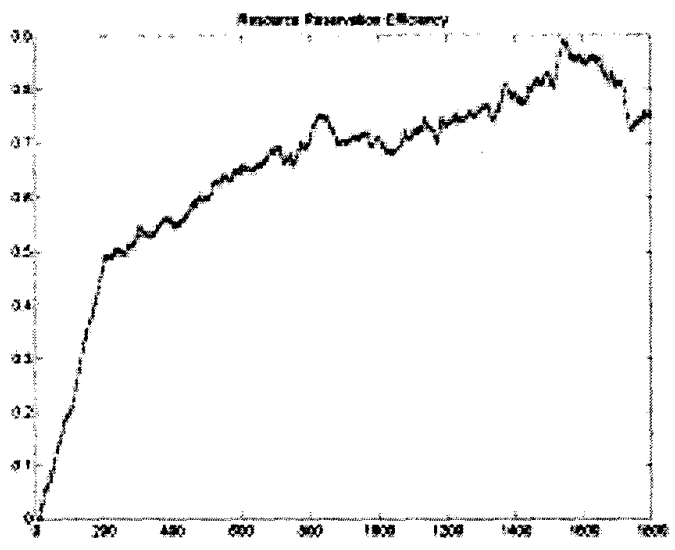

Figure 10: Resource Reservation Efficiency 